\title{
Postoperative follow up Challenge in Paediatric Cataract Surgery in Nepal
}

\section{Shrestha UD}

${ }^{1}$ Dr. Ujjowala Devi Shrestha, MBBS. MD, Assistant Professor, National Academy of Medical Sciences (NAMS), Consultant Paediatric Ophthalmologist, Tilganga Institute of Ophthalmology, Kathmandu, Nepal.

Address for correspondence: Dr. Ujjowala D Shrestha, E-mail: ujjowola@gmail.com

\begin{abstract}
Introduction: Paediatric ophthalmology is a branch of specialty care for the health of the eyes of children. It focuses on the removal of paediatric cataracts and management of refractive error, strabismus etc. Perioperative and post-operative challenges related to paediatric cataract are known to all ophthalmologists worldwide. However, in a developing country like Nepal the main challenge of these patients are the post-operative follow up. The aim of the study is to find out the ways to reduce the post-operative follow up challenges in paediatric cataract surgery. Hence the ophthalmic assistants in the community eye centers need to be trained to examine the eyes of children. Materials and Methods: This was a retrospective study. A review of patient records, operated from July 2006 to December 2007, at Tilganga Institute of Ophthalmology was done. One hundred eyes of 69 patients were operated during the study period. Results: Follow-up assessment of visual acuity was available for 19 eyes only at the end of six weeks. Conclusion: At the end of six postoperative weeks, refractive status is important. This helps to prescribe the glasses to children as per need. If the post-operative visual status is not known, it is as equal as not performing the surgery. Ophthalmic assistants at community eye centers need to be trained to evaluate and record postoperative visual status of the paediatric age group. For this regular training and refreshers course on the pediatric eye care service is being conducted at the tertiary level for the ophthalmic assistants. This is more practical in the developing countries like Nepal.
\end{abstract}

Key words: Cataract, Intraocular lens, Pediatric cataract surgery

\section{Introduction}

$\square$ aediatric cataract is one of the preventable causes of blindness in children ${ }^{1,2}$. Thakur et al did the study on surgical profile and postoperative outcomes of Pediatric cataract in the Nepalese population ${ }^{3}$. Adhikari et al did the study on Etiology and clinical profile of paediatric cataract in Tertiary Care Center of Eastern Nepal in $2007^{4}$. Paediatric cataract surgery has its clinical challenges like per-operative difficulties and postoperative visual rehabilitation. Clinical, geographical, economical challenges are more directly related with the paediatric cataract than the adult cataract. In this article, these challenges of follow up after the paediatric cataract surgery have been discussed.

A review of the paediatric cataract surgery data from July 2006 and December 2007 gives a summary of the treatment outcomes during this time and reveals efforts at Tilganga Institute of Ophthalmology to overcome challenges. Cataract surgery was performed at Tilganga Institute of Ophthalmologyunder general anesthesia. Most patients above the age of two years old undergo intraocular lens (IOL) implantation while patients less than two years old are left aphakic and receive appropriate correction for residual refraction. Followup is conducted whenever possible at Tilganga Institute of Ophthalmology. Review of our data suggests that the continued effort of Tilganga Institute of Ophthalmology is necessary to increase the post operative follow up of the patients after the paediatric cataract surgery.

\section{Materials and methods}

This was a retrospective study. A review of patient records, operated from July 2006 to December 2007, 
at Tilganga Institute of Ophthalmology (TIO) was done. Traumatic cases were excluded for this analysis because traumatic cataract was associated with injuries with anterior and posterior segment as well.

At the time of initial presentation and diagnosis of cataract, patients were registered within the TIO database and demographic information-age, gender, region of origin-was noted. Visual acuity was assessed at this time with the help of Sheridan-Gardner charts and other age-appropriate recognition acuity techniques. Cycloplegic refractions were performed using cyclopentolate for pupillary dilatation. The best corrected visual acuity was recorded. Initial examinations were performed by pediatric ophthalmologists using Kowa SL-15 portable handheld slit lamp for the anterior segment, and B-scan and indirect ophthalmoscopy with $a+20 D$ Volk lens for the posterior segment. The cataract type and the presence of associated nystagmus and strabismus was noted.

The standard pediatric cataract surgery at TIO was lens aspiration with anterior vitrectomy for those children less than two years of age. For those children above age two years, the surgical method was lens aspiration with anterior vitrectomy with IOL implantation. Polymethyl methacrylate (PMMA) lenses, manufactured at the TIO Fred Hollows IOL factory, were implanted via scleral tunnel in those cases. All pediatric surgeries were performed at TIO under general anesthesia under the care of an anesthesiologist.

Postoperatively, these children were prescribed a topical antibiotic and steroid combination drop for the first two weeks. Parents were instructed to instill these drops hourly as a means of balancing medication noncompliance with the need for adequate medication, with an actual administration rate of approximately six times daily. For the next four weeks, the medication was tapered effectively to four times a day. At the end of six weeks, children were followed up at the TIO or, if necessary, at a community eye centre or alternate eye hospital. Visual acuity was assessed in cooperative children at each post-operative visit and noted within the patient record. When possible, retinoscopy was performed six weeks post-operatively and this value was noted. Glasses were prescribed six to eight weeks post-operatively. During follow-up assessment, postoperative strabismus and complications were noted, as well as the presence of visual axis opacification [VAO] and the need for surgical capsulotomy with or without anterior vitrectomy.

\section{Results}

One hundred eyes of 69 patients were operated on at the TIO during the study period from July 2006 to December 2007. Out of these 69 cases 22 (31.9\%) were unilateral and $47(68.1 \%)$ were bilateral (Table 1).

Table 1: Laterality of eyes

\begin{tabular}{|c|c|c|}
\hline & Number & Percentage \\
\hline Unilateral & 22 & $31.9 \%$ \\
\hline Bilateral & 47 & $68.1 \%$ \\
\hline Total patients & $\mathbf{6 9}$ & $\mathbf{1 0 0} \%$ \\
\hline
\end{tabular}

Out of the 69 patients operated on, $42(60.9 \%)$ were males and $27(39.1 \%$ ) were females (Table 2 ).

Table 2: Gender distribution.

\begin{tabular}{|c|c|c|}
\hline & Number & Percentage \\
\hline Males & 42 & $60.9 \%$ \\
\hline Females & 27 & $39.1 \%$ \\
\hline Total & $\mathbf{6 9}$ & $\mathbf{1 0 0 \%}$ \\
\hline
\end{tabular}

The average age of the patients at the time of presentation was 7.4 years ( 1.1 month -15 years). Out of 100 eyes, 10 eyes were left aphakic. Lamellar cataract was most common (72/100 eyes). Of 69 patients, five had strabismus while nine had nystagmus (Table 3 ).

Table 3: Clinical presentations

\begin{tabular}{|l|c|c|}
\hline & Number & Percentage \\
\hline White pupillary reflex only & 55 & $79.7 \%$ \\
\hline Associated strabismus & 5 & $7.3 \%$ \\
\hline Associated nystagmus & 9 & $13.0 \%$ \\
\hline \multicolumn{1}{|c|}{ Total } & $\mathbf{6 9}$ & $\mathbf{1 0 0} \%$ \\
\hline
\end{tabular}

Biometry data revealed a mean axial length of $21.9 \mathrm{~mm}(16.86-28.16)(\mathrm{n}=97)$ and mean keratometry value of $43.6 \mathrm{D}(36.25-50.37)(n=98)$. The average power for implanted IOLs was 24.6 D ( $n=95)$. Followup assessment of visual acuity was available only in 19 eyes. Four eyes had $6 / 6$ to $6 / 9$, six eyes had $6 / 12-6 / 18$, six eyes had $6 / 24-6 / 36$, and three eyes had $<=6 / 60$ (Table 4 and Fig1).

Table 4: Post-operative visual acuity.

\begin{tabular}{|l|c|c|}
\hline & Number & Percentage \\
\hline $6 / 6$ to $6 / 9$, & 4 & $21 \%$ \\
\hline $6 / 12-6 / 18$ & 6 & $31.6 \%$ \\
\hline $6 / 24-6 / 36$ & 6 & $31.6 \%$ \\
\hline$<=6 / 60$. & 3 & $15.8 \%$ \\
\hline \multicolumn{1}{|c|}{ Total } & 19 & $100 \%$ \\
\hline
\end{tabular}




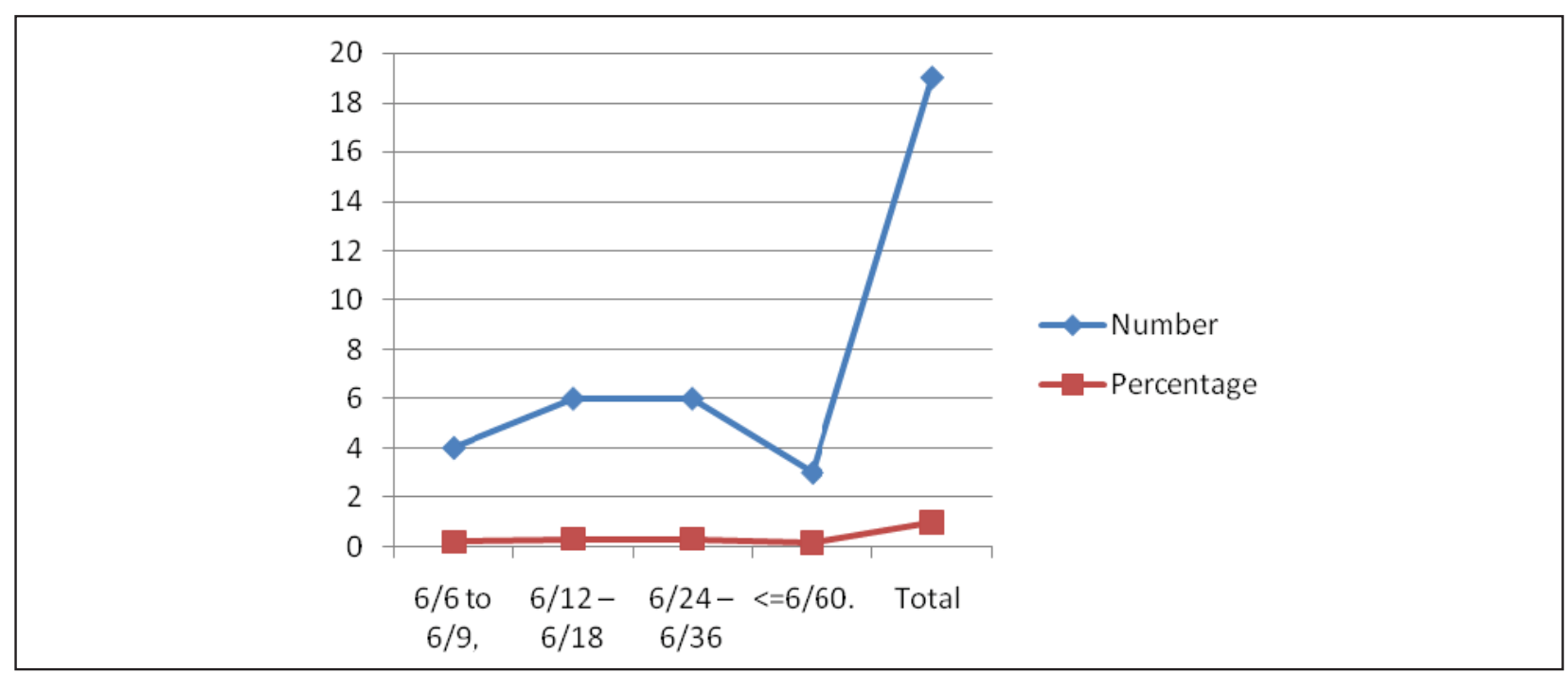

Fig 1: Post-operative visual acuity

Follow-up assessment at six weeks revealed 2 eyes with pupillary capture and one eye with IOL decentration. Visual axis opacification (VAO) was observed in 1 eye requiring a surgical intervention. The minimum duration of the follow up was one week and maximum duration of the follow up was six weeks.

\section{Discussion}

The management of paediatric cataract is paediatric cataract surgery. As IOL implantation has increasingly been supported as standard of care in the setting of cataract in children beyond their infancy, this procedure has become more common in Nepalese practice as well ${ }^{1,2}$. However, this is the start of the management. The good visual outcome depends on the regular follow up of the patients after the pediatric cataract surgery.

Certain unique challenges are present in the health service delivery in Nepal. First, Nepal's topography makes development of any health care infrastructure in the more mountainous northern regions very difficult ${ }^{5,6,7}$. Most of the eye hospitals in Nepal are situated in the southern region of Nepal, predominately in the Terai region along the Indian border. Even though community ophthalmic services and school-based eye screening have complemented paediatric eye care through early detection, counseling and referral, most of the villages are very far from access to hospital care and transportation inhibits timely care of paediatric cataracts. This is the cause for the late presentations in our study. The average age of the patients at the time of presentation was 7.4 years (1.1 month - 15 years). Follow-up assessment of visual acuity was available for 19 eyes only out of the 100 eyes.. Four eyes had $6 / 6$ to $6 / 9,6$ eyes had $6 / 12-6 / 18,6$ eyes had $6 / 24-6 / 36$, and 3 eyes had $<=6 / 60$. This is because of the poor follow up of the patients after the paediatric cataract surgery.

Second, low literacy rates in the Nepalese population (estimated in 2005 as less than half of the adult population) significantly limits general awareness about congenital and juvenile cataracts and the appropriate treatment. The general population knows little about the implications, prognosis, and availability of treatment for cataracts in children. The people in general think that "The surgery" is the treatment. Thus to increase the awareness the local ophthalmic technicians are trained to encourage the parents to seek eye care services within 6 weeks after birth. They are taught about the importance of post-operative medication after the paediatric cataract surgery. The ophthalmic technicians are trained to do the refraction of children.

The importance of appropriate care and the postoperative visits in this setting cannot be overemphasized: paediatric cataract is the leading cause of treatable childhood blindness in the world, and there are around 190,000 blind children suffering from cataract worldwide. The number of "blind years" in children is much greater than when blindness occurs in adulthood from cataract. Accordingly, quality of many years of life is improved drastically by operating well-selected paediatric cataract cases and timely post-operative follow up. However, if the refractive status of the patient is not known, it is as equal as not performing the surgery in those patients.

The global challenges include financial constraints on families, limited available technology, and sparse follow-up. TIO has sought to overcome these formidable challenges within the Nepalese context. Paediatric eye care resources are focused at its tertiary center. TIO uses its eye care network to make appropriate paediatrics 
referrals. The operated children are followed-up at community eye centers for refraction whenever feasible.

Nepal continues to experience imbalance in the health workforces due to shortage of personnel and geographical maldistribution ${ }^{9}$. Shortages are especially severe in rural areas, since health professionals are often concentrated in cities ${ }^{10}$. Increased health care worker emigration is contributing to a shortage of trained health professional in Nepal. Currently, Nepal has only eight paediatric ophthalmologists for a total population of over 20 million, with over 40 percent of the population under the age of 15 . This works out to a ratio so low as to inevitably provide a significant barrier to care for most Nepalese children. Hence this ratio can be tackled by training of the human resources like the ophthalmic technicians to take care of the children in primary level. There are no quick-fixes to overcome the above challenges ${ }^{8}$.

This network of ophthalmologic care center is one of the foundational elements of TIO's strategy for providing treatment that is specific to the Nepalese context as a landlocked country with terrain that significantly limits health care access in more remote regions of the country. This network has to be intensified.

\section{Conclusion.}

The management of pediatric cataract is pediatric cataract surgery. As IOL implantation has increasingly been supported as standard of care in the setting of cataract in children beyond their infancy, this procedure has become more common in Nepalese practice as well. However, this is the start of the management. Postoperative follow up of pediatric cataract remains one of the most difficult challenges. Hence the post operative visual acuity of the children operated for paediatric cataract cannot be recorded. The limitation for the pediatric ophthalmologists is that paediatric cataract surgery needs to be done although the patients may not come for the follow up. To increase follow up, ophthalmic assistants at community eye centers (CEC) needs to be trained to evaluate and record postoperative outcomes. For this, regular training and refreshers course on the paediatric eye care service needs to be conducted more at the tertiary level for the ophthalmic assistants.

Acknowledgements: None.

Funding: None.
Conflict of Interest: Nil.

Permission from IRB: Yes.

\section{References}

1. Wilson ME Jr, Bartholomew LR, Trivedi RH. Pediatric cataract surgery and intraocular lens implantation: practice styles and preferences of the 2001 ASCRS and AAPOS memberships. J Cataract Refract Surg 2003;29:1811-20.

2. Wilson ME Jr, Trivedi RH, Buckley EG, Granet DB, Lambert SR, Plager DA, Sinskey RM, Vasavada AR. ASCRS white paper. Hydrophobic acrylic intraocular lenses in children. J Cataract Refract Surg 2007;33:1966-73.

3. Thakur J, Reddy H, Wilson ME Jr, Paudyal G, Gurung R, Thapa S, Tabin G, Ruit S. Pediatric cataract surgery in Nepal. J Cataract Refract Surg 2004;30:1629-35.

4. Adhikari S, Badhu BP, Bhatta NK, Jha CB, Baral N, Kumari N. Etiology and clinical profile of pediatric cataract in a tertiary care center of Eastern Nepal. $J$ Nepal Med Assoc 2007;46:94-8.

5. Sharma, B. Utilisation of Antenatal Care Services in Nepal. Nepal Population Journal 2004;11(10):7997.

6. UNFPA, (2005) Nepal Conflict Aggravates Women's Reproductive Health Risks. Press release, 30 November, Available at http://www.unfpa.org/news/ news.cfm?ID=7 21, United Nations Population Fund.

7. Matsumura, M, Gubhaju B. Women's Status Household Structure and the Utilisation of Maternal Health Services in Nepal. Asia-Pacific Population Journal. 2001;16(1):23-44.

8. Simkhada B, van Teijlingen ER, Porter M, Simkhada P. Major problems and key issues in Maternal Health in Nepal. Kathmandu Univ Med J 2006;4:258-63.

9. WHO (2004) Country Health Profile Nepal. World Health Organization, Regional Office for SouthEast Asia.

10. Safe motherhood (1998) Skill care during child birth. Available at http://www.safemotherhood.org/ smpriorities/index.html. Accessed on 02-12-2005.

How to cite this article?

Shrestha UD. Postoperative follow up Challenge in Paediatric Cataract Surgery in Nepal. J Nepal Paediatr Soc 2011;31(3):198-201. 